\title{
Concept Design of Headset Development Project
}

\author{
Liu Guo-qing
}

\author{
Wenzhou Vocational \& Technical College, Wenzhou. 325035 \\ 3685424@qq.com
}

\begin{abstract}
Keywords: Project;Headset;Development;Demand;Design.
\end{abstract}
\begin{abstract}
A headset which is convenient to carry may eliminate the cumbersome of traditional headset with lines through USB connection. Compared with traditional one, it can be used in various environments like video chat and listening to music during travel. In nowadays, many people like to play games and they prefer a headset with better sound effect which is also convenient to carry. While many people do not use because of too large headset or mess wires, and such headset is very suitable for them because it is convenient and do not occupy much space, and it is very popular among many people who often go out.
\end{abstract}

\section{Introduction}

Most of the existing headset all have lines and some wireless headsets are blue-tooth or radio which do not suit computer at all, such issue exists in both domestic and aboard. At the same time, most headsets are folding for easy carrying, while such headsets usually have lines and you will fell confused after wrapping. After taking initial market research as well as user research, I still do not know what I should do, I make the schemes and overturn one by one. Finally, I find that we can combine the wireless device which is suitable for computer and the expansion characteristics of McCarthy together. The ear pack basically uses black to increase the mechanical sense. In addition, I use silver metal for McCarthy and make the whole headset look more cool and more appropriate to young people. The main reason I modify the scheme time and time again is that design is more than just a picture, we have to design within various constraints through combining with manufacture as well as technological level we may achieve now, this is the most important ability for a designer.

\section{Research background}

After 30 years of reform and opening up, Chinese economic level has been greatly increased than ever before and become the third largest economy in the world. What is more, it has even reached the level of moderately developed countries. As a moderately developed country, Chinese technical level also ranks in front of the world. Computer is essential in the information society. Similarly, computer accessories are also very important. Many people deem that a keyboard and mouse are enough for computer, and a power supply is enough for notebook, but in fact it is not true. One main reason they think so is that they dislike the complex of other parts. For example: they usually do not want to take the keyboard and mouse when carrying notebook. Keyboard really can be omitted, but it is not very inconvenient for them to touch the keyboard with their hands. They feel cumbersome to take the mouse, as a result, wireless mouse emerges. Similarly, many people do not like to carry their headset because it has longer lines and larger space which is more complex that mouse. In fact, people still like to use the easy carrying headset that occupies a small place, so it is imminent to design such a convenient and dis-cumbersome headset.

\subsection{Domestic and foreign development situation}

Compared with headset development aboard, domestic headset still has some shortcomings, there are some domestic well-known headset brands like DUNU. If starting simply from the sound quality, DUNU is quite a good choice for ordinary consumers. It used to be a Taiwan industry and OEM was better. Later it stationed in the mainland and the sound quality of its headset is relatively good such as: 
DN-EX01 and S01 which is earlier sightly. Of course, all these are not enough to compete with foreign industry.

Edifier is also a famous brand in domestic. For example: H260 has a heavy bass and clear high voice. Different from other brands that mainly focus on bass ear-bud, its high voice is quite clear.

Headset industry has not received enough attention in domestic, as a result, domestic headset industry lags far behind foreign headset industry. Domestic headset usually has no focus, the sound effect is quite good when listening to the music, but it still cannot reach the requirements of high-end headset. This design aims at improving the disadvantages of domestic headset

\subsection{Main design contents}

Main design contents of portable headset:

1) Complete market survey, information retrieval, patent search and other works.

2) Complete sketch schemes 1-4 and as shown in figure 1, complete different sketch effects according to different conditions.

3) Overall design scheme and concrete structure of portable headset.

4) McCarthy and receiver coil design of portable headset.
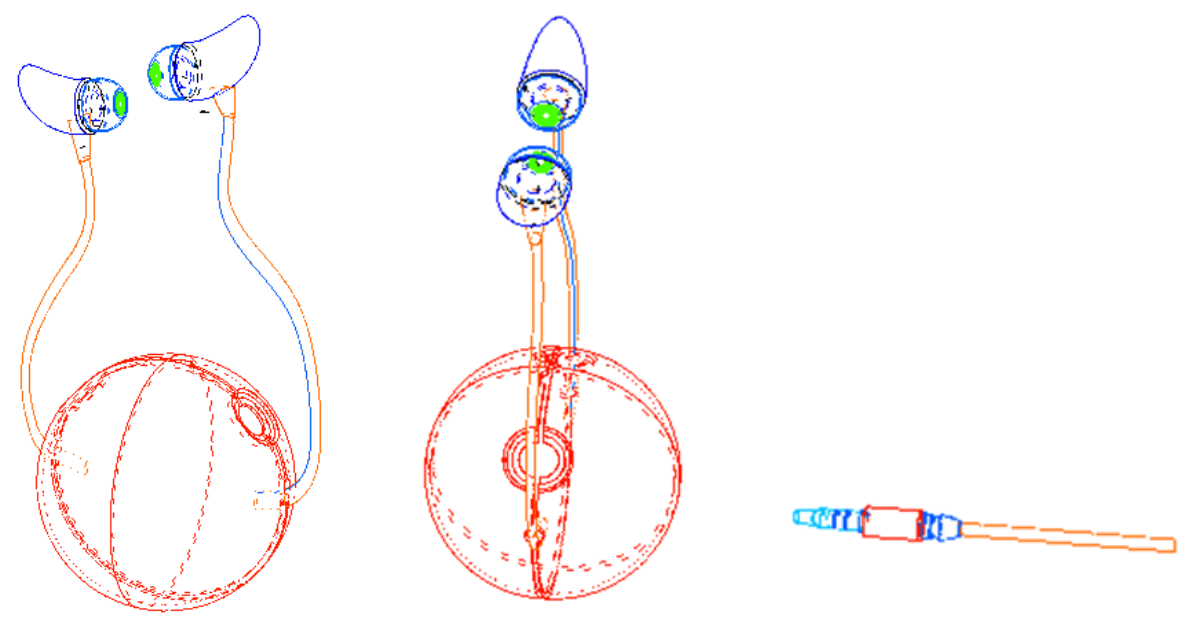

Figure 1 Sketch scheme

\section{Overall design scheme}

\subsection{Modeling conceptual design of McCarthy}

As shown in figure 2, McCarthy is divided into seven small parts with distinct levels. It achieves the purpose of expansion through using such ladder shapes and narrows the size of entire McCarthy as big as middle part. As a result, space occupied by the headset decreases and it can be stored in a more regular shape when not using. Rotary joints on both sides of McCarthy achieve better agreement between headset and ears and make ears more comfortable. In addition, such ladder shape will present a sense of machine when stretching and become more popular among students. 


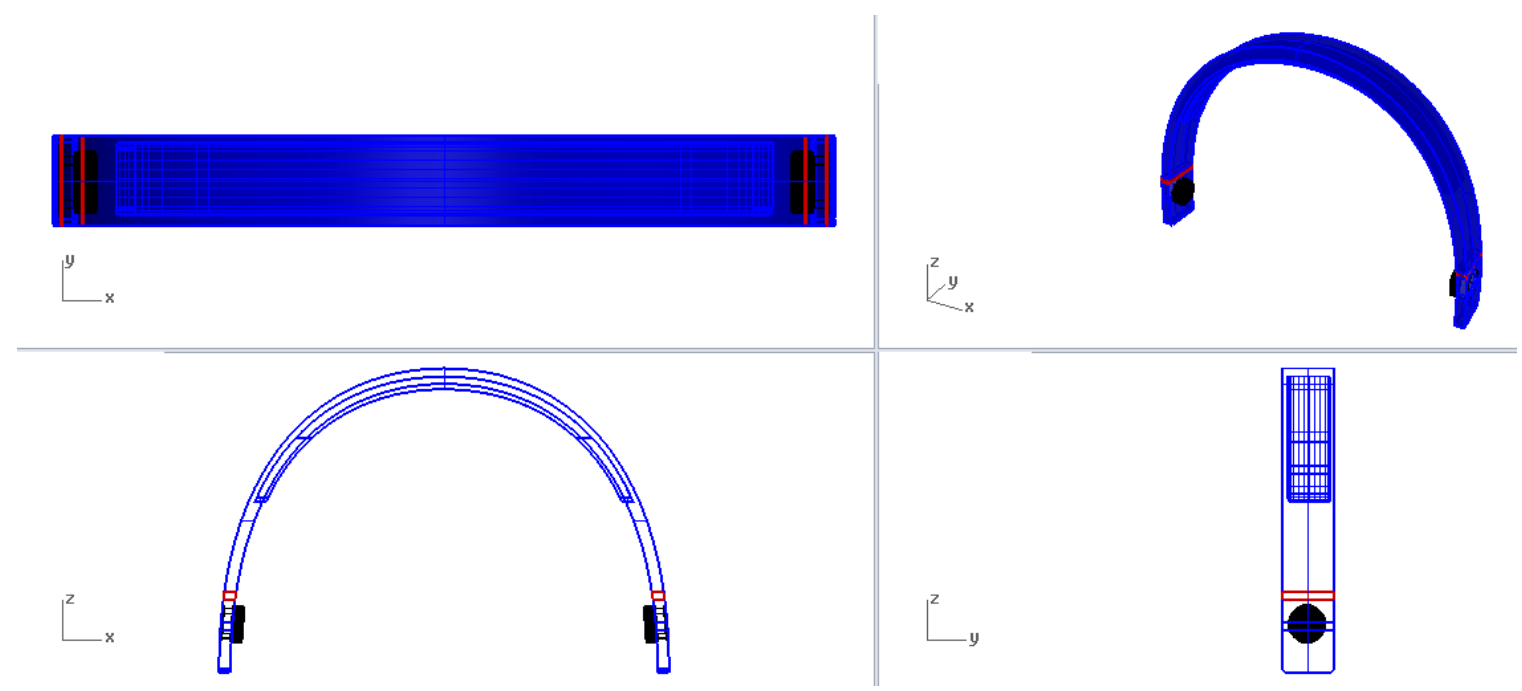

Figure 2 Unfolding three orthographic view

\subsubsection{Conceptual design of ear pack}

As shown in figure 3, the receiver is covered with thicker cotton ring because such cotton ring is relatively thick which makes ears more comfortable when using. In addition, the ear pack is relatively large which can better cover ears, the sound effect can be better without disturbing other people. Our ears will not feel pain after wearing for a long time, and the two receivers will merge together when McCarthy shrinking. This thick cotton ring may add a buffer and prevent direct connection as well as extrusion, and then increase the service life of headset.

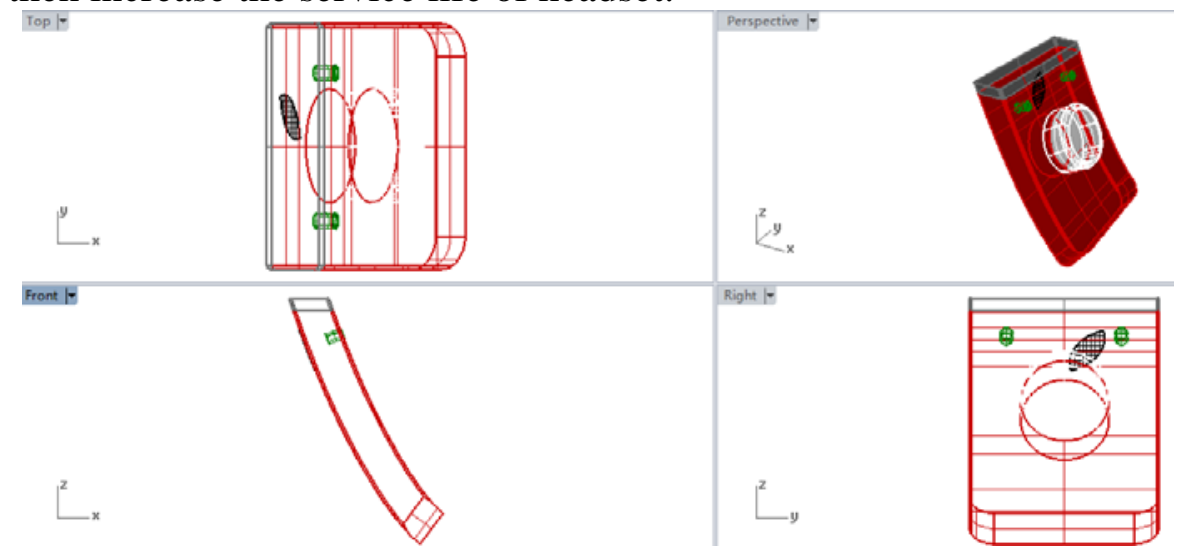

\subsubsection{USB conceptual design}

Figure 3 Three orthographic view of ear pack

Microphone design of this product gets rid of the swing type microphone and there is a fixed one under the receiver. Microphone is designed to be very small and it almost does not occupy space for convenient carrying. While it will not weaken the speech effect, and on the other hand, receiving intensity of such microphone is relatively huge, we can clearly say what we want to express without loud speaking. Apart from these, the listening will be very clear too and it has no effect on speaking if the mouth was far away. USB design aims at getting rid of the winding trouble of traditional cable ones which often look very messy because the wire must be wound up. Design of this USB solves this problem, USB device is just placed on one side of the headset, we may pull it out when not using, it is easy to understand. In addition, it is not easy to lose because we have to place it back on the headset.

\subsection{Working principle of headset}

The working principle of wireless headset mainly transfers signal through blue-tooth technology.

Using digital radio frequency may provide more adequate broadband for short distance communication. As long as within limited distance, high radio frequency can be used in any place without affections from obstacles. Transmission distance can reach 10 to 20 meters in general which is enough for most users. The biggest feature of wireless is that it achieves radio frequency remote control from 360 degrees. In addition, the power consumption of wireless devices is relatively low, and it also has a dormant state of working standby. Receiving end of the wireless device is usually 
built-in receiver and transmitter is installed on the host device, and such design has no effect on product appearance.

The wireless receiver itself has double or multiple bands, and then multiple wireless devices may conduct management work through this receiver. It will not interact with other wireless devices when working.

\subsection{Basic parameters}

Table 1

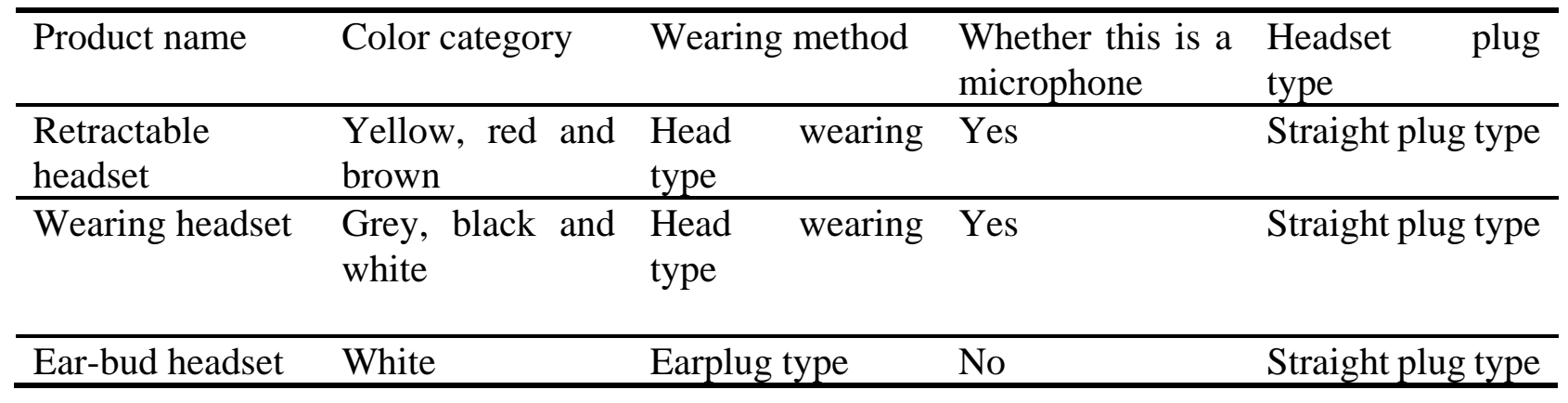

\section{Selection of production materials}

\subsection{Material selection for McCarthy}

McCarthy selects engineering plastics to achieve smooth appearance as well as certain toughness and stress. This material has no stiff feeling and the workmanship is pretty fine. In addition, the feeling is wonderful in contrast and coupled with silver appearance, there is a sense of metal which is attractive.

\subsection{Material selection for earpiece ring}

Material selection for earpiece ring is cashmere fabric. This is an enclosed design and can protect the earpiece and ears better, in addition, the voice is more clarity.

\subsection{Material selection for earpiece shell}

Material selection for earpiece shell is hard plastic which is not easy to deform. It is used to fix the appearance and protect the earpiece from outside, and it is also suitable for USB device.

\section{Summary}

Although the design of this headset is suitable for most people, the main target crowd are the youth, especially students. Black-oriented handset and silver-oriented McCarthy present the heavy and cool sense of headset which will attract the attention of students in particular. The thick earpiece ring not only reduces sound leakage, but also comforts your ears which is suitable for long time use. For example: now many college students all use computers for a long time and they need such a comfortable headset. In addition, they also need a easy carrying headset when taking a notebook outside, and at this time, such comfortable and easy carrying headset will become the first choice.

\section{References}

[1] YUAN Zhi-bin, REN Zhong-bao.Application and Thinking of Delphy in Technology Foresight[J].Science and Technology Management Research， 2006 (10） : 88-93.

[2] YU Qiu-yu.What is Culture[M].Wuhan: Changjiang Literature and Art Press, 2012.

[3] SUH Ki-Soo , LEE You ng Eun .Th e Rol e of Vi rtual Experience in Consum er Learning :An Em perical Invest igation [ J] .MIS Qu artly , 2005 , 29(4):115 -143 .

[4] LE E S ang-yoon, CHEN Ti an , KIM Jong-seo, et al.Using Vi rtual Reali ty for Evalu at ing Af f ective Propert ies of Product Design [ C] .USA :IE EE V R 2004 Conf erence, 2004. 